\title{
Esophagitis Dessicans Superficialis - Impressive Endoscopic Appearance of a Benign Condition
}

\author{
Syed Adnan Mohiuddin ${ }^{1 *}$, Rafie Yakoob ${ }^{2}$, Hamidullah Wani ${ }^{2}$, Omer Eljyli Elssedig ${ }^{2}$, Khalid Mohsen Al \\ Ejji $^{2}$ and Saad Rashid Al Kaabi ${ }^{2}$
}

${ }^{1}$ Department of Endoscopy, Hamad Medical Corporation, Qatar

${ }^{2}$ Department of Gastroenterology, Hamad Medical Corporation, Qatar

Received: December 12, 2017; Published: December 19, 2017

*Corresponding author: Syed Adnan Mohiuddin, Clinical Fellow, Department of Endoscopy, Hamad Medical Corporation, Doha, P0 B0X 3050, Qatar

\begin{abstract}
Esophagitis Dessicans Superficialis (EDS) refers to a rare and unique lesion of the esophagus characterized by sloughing of esophageal mucosa. A benign condition, it is thought to be a mucosal reaction to various types of insults or a mucosal allergic response. Various clinical presentations, endoscopic appearances and associations with systemic diseases have been described in literature. We report 4 consecutive cases of this rare condition in our endoscopy practice with varying endoscopic characteristics and present a literature review of EDS.
\end{abstract}

Abbreviations: EDS: Esophagitis Dessicans Superficialis; EGD: Esophagus Gastro duodenoscopy; PAS: Periodic Acid Schiff; HSV: Herpes Simplex Virus; CMV: Cytomegalovirus; PPI: Proton Pump Inhibitor Therapy; SLE: Systemic Lupus Erythematosus

Cases

\section{Case 1}

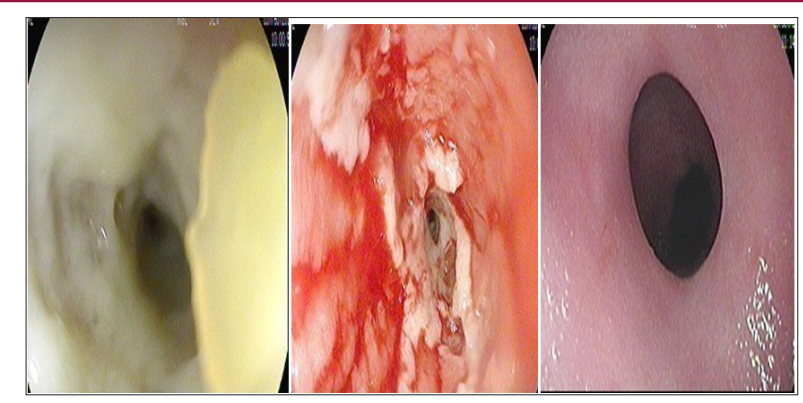

Figure 1:

15-year-old male diagnosed with cerebral palsy and seizure disorder was referred for dysphasia to solid food progressing over 3 months to dysphasia to both solids and liquids. His medications included Lamotrigine for his Seizure disorder, Supplemental Iron for Iron deficiency anemia and Lansoprazole once daily for reflux disease. Esophagus gastro duodenoscopy (EGD) showed that the lower third of the esophagus has yellowish plaque like material (Figure 1a) which on removal revealed sloughed and friable mucosa (Figure 1b) and a partial stricture at the distal esophagus. A $9 \mathrm{~mm}$ scope could not pass the distal esophagus due to a partial stricture but a $5 \mathrm{~mm}$ slim scope was passed. Histopathology showed esophageal mucosa with extensive ulceration and granulation tissue. There were no viral inclusions, granuloma, dysplasia or malignancy.
Staining for Periodic Acid Schiff (PAS), Herpes Simplex Virus (HSV) and Cytomegalovirus (CMV) was negative. He was prescribed twice daily dose of Proton pump inhibitor therapy (PPI) for 2 months. Upon clinical improvement the patient was prescribed once daily PPI therapy for the next 4 months. Repeat OGD after 6 months of PPI therapy showed a normal and completely healed esophageal mucosa with no evidence of the previously noted partial stricture. (Figure 1c).

\section{Case 2}

35-year-old male known to have colon cancer status post total hemicolectomy and on chemotherapy was referred due to persistent dysphasia and recurrent vomiting. Medication history was significant for chemotherapy regimen Folfiri Cetuximab with his last cycle received two weeks back. OGD revealed sloughed lower esophageal mucosa with whitish cast like material adherent to it (Figure 2a). After flushing the casts with water jet the underlying mucosa showed ulcerations and sloughing (Figure 2b). Histopathology revealed ulceration and inflammation with no granuloma, metaplasia or dysplasia. Stains for CMV and HSV were negative. His chemotherapy was continued and a prescription of twice daily PPI therapy was added. After 4 months the patient had a repeat OGD that showed complete healing and normal esophageal mucosa except for a small hiatus hernia (Figure 2c). The dysphasia and vomiting subsided with mild residual reflux symptoms at 6 months. 


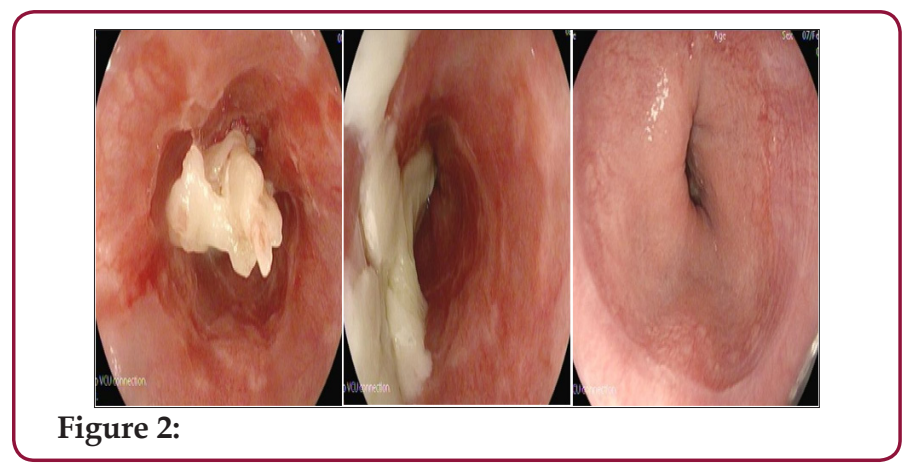

\section{Case 3}

55 y/o male known to have diabetes and chronic reflux symptoms not responding to PPI once daily were referred for endoscopy. His medications included metformin, glimepiride, sitagliptin, aspirin and esomeprazole. OGD was remarkable for whitish membranes at lower esophagus resembling 'gift wrap ribbons' suggestive of esophagitis Dessicans Superficialis (Figures 3a \& 3b). Histopathology showed inflammation without evidence of granuloma, dysplasia or malignancy. Staining for PAS, HSV CMV was negative. The patient was maintained on once daily PPI therapy. $\mathrm{He}$ had minimal reflux symptoms on his follow up 1 year post first presentation. A repeat OGD was not pursued in the patient.
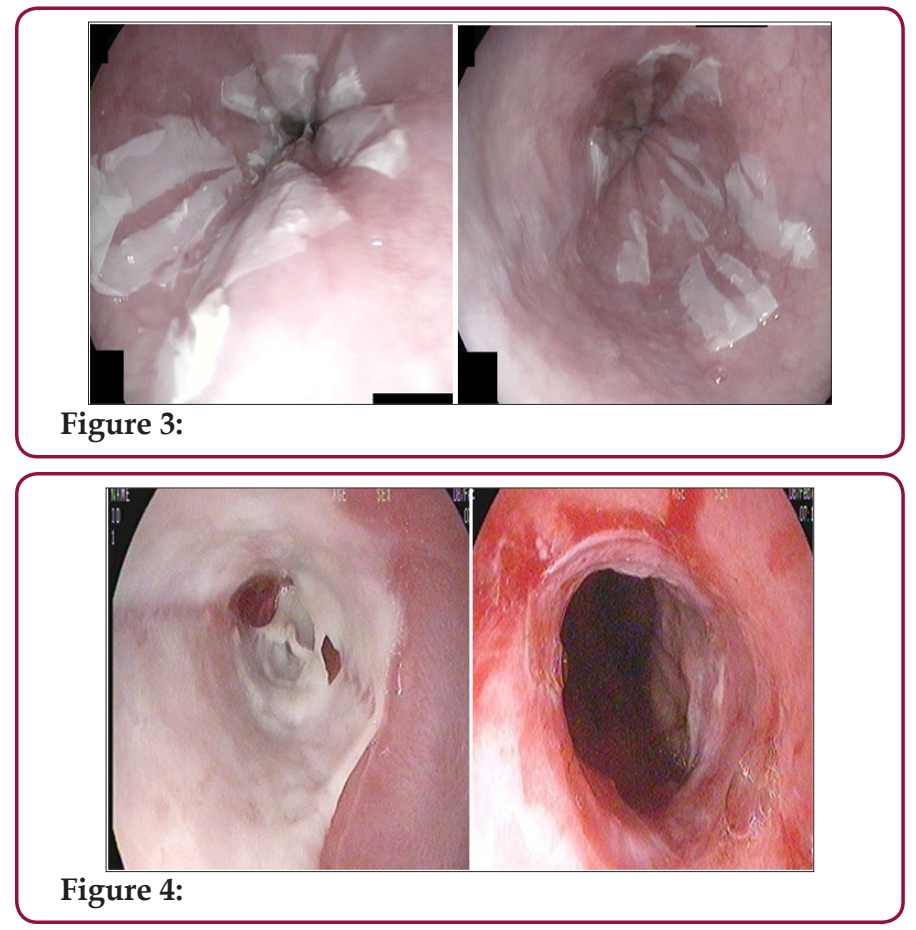

\section{Case 4}

An 80 y/o female with background of end stage renal disease on hemodialysis, diabetes and hypertension presented with hematemesis. Her medication history included multiple medications including Carvidilol, Hydralazine, Amlodipine, Pantoprazole, along with iron and phosphate supplementation. OGD showed whitish membranes sticking to the lower esophagus (Figure 4a). When washed and removed with water jet underlying sloughing of mucosa at lower end of esophagus was observed (Figure 4b). Histopathology of the esophageal mucosa showed inflammatory and necrotic debris with fibrin, suggestive of sloughing esophagitis. There was no evidence of granuloma, dysplasia or malignancy. PAS stain for fungi was negative. She was prescribed twice daily PPI therapy. The patient did not suffer from any more hematemesis or vomiting after 2 weeks. A repeat endoscopy to assess mucosal healing was not pursued at that stage due to her advanced age.

\section{Discussion}

Esophagitis Dessicans Superficialis (EDS), also referred to as Sloughing Esophagitis in literature is an endoscopic diagnosis characterized by sloughing of the esophageal mucosa and overlying casts or membranes. Various clinical presentations, endoscopic appearances and associations with systemic diseases have been described in previous case reports. We provide a literature review from such previous reports and compare it with the cases described in the current series.

Associations: EDS has been thought to be idiopathic and unexplained in most recognized cases [1]. Few case reports have suggested strong association with desquamating esophageal disorders, in particular Pemphigus Vulgaris [2,3]. Rao at el evaluated 42 patients with vesciculobullous dermatosis and found esophageal involvement in 27 patients (67\%) and classical appearance of EDS in 2 patients (5\%) [4]. other reports have described its association with bullous Systemic Lupus Erythematosus (SLE) [5] and with celiac disease [6]. Is also believed that EDS occurs in older population on multiple medications. Purdy et al evaluated thirtyone patients with endoscopic appearance of necrotic superficial squamous epithelium, with endoscopic appearance of white plaques or membranes i.e. sloughing esophagitis [7].

Compared with controls these patients were older and more likely to be taking five or more medications. No particular cause could be identified for the condition but the authors concluded stasis and contact injury due to multiple medications may lead to the condition. Other case reports have described associations with medications, typically bisphosphonates [8] and post sclerotherapy of varices [9]. One of the four patients we describe here was over 60 year's age and one was female. None of our four patients suffered from vesciculo bullous disease. Pre-existent co morbid conditions was a consistent factor among all the cases - Cerebral Palsy in case 1; GI malignancy post treatment in case 2; Diabetes Mellitus in case 3,4 and ESRD in case 4 All patient in the series were on multiple medications. Two of our patients were on oral iron therapy (cases 1,4). Three of the four patients (Case 2,3,4) were on five or more medications as described by Purdy et al. [7].

Clinical Presentation: Patients with EDs may present with a variety of symptoms ranging from dysphasia - odynophagia to more dramatic presentations of vomiting of casts (1). The variety of presentations in our case series is diverse - dysphasia in cases 1 and 2 , chronic reflux in case 3 , vomiting in cases 2 and 3, hematemesis in case 4.

Endoscopic Appearance: Endoscopic appearance of EDS is typically described as peeling of esophageal mucosa with linear plaques or membranes $[1,10]$ or vertical strips of sloughing 
esophageal mucosa creating a remarkable 'Gift Wrap Ribbons' appearance [11]. Yellowish material causing luminal occlusion, which on removal revealed extensive sloughing of underlying mucosa has also been, described [12]. Our cases too have varied endoscopic appearances - yellowish plaques in cases 1, whitish plaques in 2, whitish membranes in cases 3 and 4 . One of our cases had a classical gift wrap ribbon (Case 3) appearance on endoscopy. A partial stricture was observed in case 1. Histopathological exam shows sloughing and flaking of superficial squamous epithelium with occasional bullous separation of the layers, parakeratosis and varying degrees of acute or chronic inflammation [1]. Histopathology has not been well described [10], but may be more useful to exclude other differentials of sloughing esophagitis like fungal or viral infections and Eosinophilic esophagitis.

Differential Diagnosis: includes conditions that cause endoscopic appearance of sloughing of the esophageal mucosa like Eosinophilic esophagitis, Corrosive injury of the esophagus and infections of viral or fungal etiology.

Management: Inspite of the impressive endoscopic appearance EDS remains a benign condition without lasting pathology [1]. Acid suppression and discontinuation of any precipitins factors can help in mucosal healing. Steroids are needed if associated with bullous dermatosis. Indeed, all our patients responded symptomatically to acid suppression with PPI. Complete endoscopic healing was demonstrated in two cases (case 1 and 2). No comment could be made on endoscopic healing in cases 3 and 4 due to lack of a repeat endoscopy. Symptomatic response was seen in all of our patients to 3 to 6 month courses of PPI.

\section{Conclusion}

a) EDS is a benign condition that can occur at any age with varying clinical presentation related to upper GI tract.

b) EDS is usually associated with an underlying co morbid illness. Patients on multiple medications are at increased risk.

c) Diagnosis is made endoscopic ally with findings of yellowish or whitish plaques or membranes with underlying sloughed mucosa.

d) Histopathology is needed to rule out other etiologies of similar endoscopic findings. e) EDS responds to correcting the underlying condition, reducing the number or offending drugs and acid suppression with proton pump inhibitor therapy.

\section{References}

1. Carmack SW, Vemulapalli R, Spechler SJ, Genta RM (2009) Esophagitis dissecans superficialis ("sloughing esophagitis"): a clinicopathologic study of 12 cases. Am J Surg Pathol 33(12): 1789-1794.

2. Cesar WG, Barrios MM, Maruta CW, Aoki V, Santi GG (2009) Oesophagitis dissecans superficialis: an acute, benign phenomenon associated with pemphigus vulgaris. Clin Exp Dermatol 34(8): e614-616.

3. Hokama A, Yamamoto Y, Taira K, Nakamura M, Kobashigawa C, et al. (2010) Esophagitis dissecans superficialis and autoimmune bullous dermatoses: A review. World Journal of Gastrointestinal Endoscopy 2(7): 252-256.

4. Rao PN, Samarth A, Aurangabadkar SJ, Pratap B, Lakshmi TS, et al. (2006) Study of upper gastrointestinal tract involvement in pemphigus by esophago-gastro-duodenoscopy. Indian J Dermatol Venereol Leprol 72(6): 421-424.

5. Yogarajah M, Sivasambu B, Jaffe EA (2015) Bullous Systemic Lupus Erythematosus Associated with Esophagitis Dissecans Superficialis. Case Reports in Rheumatology.

6. Hage-Nassar G, Rotterdam H, Frank D, Green PH (2003) Esophagitis dissecans superficialis associated with celiac disease. Gastrointest Endosc 57(1): 140-141.

7. Purdy JK, Appelman HD, McKenna BJ (2012) Sloughing esophagitis is associated with chronic debilitation and medications that injure the esophageal mucosa. Mod Pathol 25(5): 767-775.

8. Hokama A, Ihama Y, Nakamoto M, Kinjo N, Kinjo F, et al. (2007) Esophagitis dissecans Superficialis associated with bisphosphonates. Endoscopy 39(S1): E91.

9. Perez-Carreras M, Castellano G, Colina F, Rodríguez-Muñoz S, SolísHerruzo JA (1998) Esophagitis dissecans Superficialis (esophageal cast) complicating esophageal sclerotherapy. Am J Gastroenterol 93(4): 655656.

10. Akhondi H (2014) Sloughing esophagitis: a not so common entity. Int J Biomed Sci 10(4): 282-286.

11. Brownschidle SS, Eric K Ganguly, Rebecca L Wilcox (2014) Identification of Esophagitis Dissecans Superficialis by Endoscopy. Clinical Gastroenterology and Hepatology 12(9): e79-e80.

12. Costa Santos V, Filipa Ávila, Paulo Massinha, Nuno Nunes, Ana Catarina Rego, et al. (2015) Sloughing Esophagitis: A Rare Cause of Dysphagia. GE Portugese Journal of Gastroenterology 22(1): 37-38.

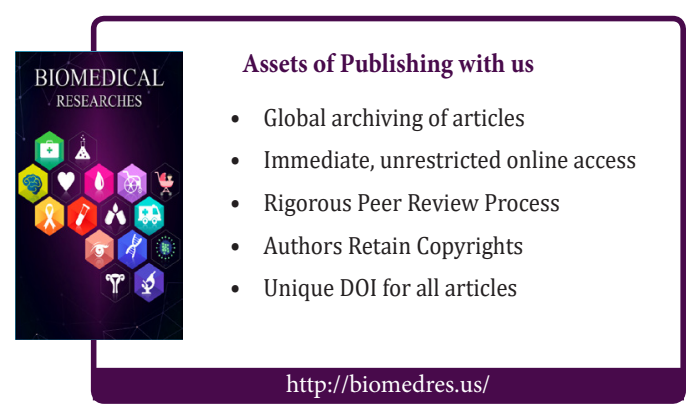

